\title{
UNIFORM APPROXIMATION ON UNBOUNDED SETS BY HARMONIC FUNCTIONS WITH LOGARITHMIC SINGULARITIES ${ }^{1}$
}

\author{
BY
}

P. M. GAUTHIER, M. GOLDSTEIN AND W. H. OW

\begin{abstract}
This paper deals with the qualitative theory of uniform approximation by harmonic functions. The theorems of Brelot and Deny on Runge- and Walshtype approximation on compact sets are extended to unbounded closed sets.
\end{abstract}

1. Introduction. Suppose $h$ is harmonic in a deleted neighborhood of a finite point $a \in \mathbf{R}^{2}$. We say that $h$ has a logarithmic singularity at $a$ if there is a function $u$ harmonic at $a$, and a constant $\alpha$ such that in a neighborhood of $a, h-u$ is of the form

$$
\alpha \ln |z-a| \text {. }
$$

Since this notion is conformally invariant, it also makes sense on a Riemann surface $R$. An essentially harmonic function on $R$ is a function which is harmonic except possibly for logarithmic singularities. It follows from Lemma 4 below that an essentially harmonic function can be written as the difference of subharmonic functions.

Without loss of generality we may and shall assume that every Riemann surface $R$ is connected. Following Scheinberg [12] we say that a subset is bounded in $R$ if its closure in $R$ is compact. A Riemann surface $R^{\prime}$ is said to be an extension of $R$ if $R$ is (conformally equivalent to) an open subset of $R^{\prime}$. If furthermore $\bar{R} \neq R^{\prime}, R^{\prime}$ is an essential extension of $R$. We shall say that a closed subset of $R$ is essentially of finite genus if $F$ has a covering by a family of pairwise disjoint open sets, each of finite genus. We may assume that such a cover is locally finite.

Throughout this paper, $R$ denotes an open Riemann surface and $R^{*}$ its one point compactification. Unless otherwise specified, all topological notions refer to $R$. Thus, if $F$ is a subset of $R, F^{-}$denotes the $R$-closure of $F, \partial F$ is the $R$-boundary, etc.

In this paper we establish the following results:

THEOREM 1. Let $F$ be closed and essentially of finite genus in an open Riemann surface $R$. Then, each function essentially harmonic on $F$ is the uniform limit of functions essentially harmonic on $R$.

Received by the editors May 8, 1979 and, in revised form, September 4, 1979. Presented at the conference in Classical Complex Analysis, Purdue University, 1980.

1980 Mathematics Subject Classification. Primary 30E10, 30F15, 31 A05.

${ }^{1}$ Research supported in part by N.S.F. grants MC 576-82924 and MSC 77-00962, by the N.R.C. of Canada, and by the Ministère d'Education du Québec. 
Let us call the approximation in Theorem 1 a Runge-type approximation by essentially harmonic functions. A Runge-type approximation by harmonic functions is defined analogously by replacing essentially harmonic functions by harmonic functions. Denote by $\hat{F}$ the union of $F$ and all bounded components of $R \backslash F$.

THEOREM 2. Let $F$ be closed in an open Riemann surface $R$. The following conditions are necessary in order for a Runge-type approximation by harmonic functions to be possible on $F$.

(I) $R^{*} \backslash \hat{F}$ is locally connected.

(II) For each bounded open set $V$ such that $\partial V \subset F$, either $V \subset F$ or $V \cap F=\varnothing$.

(III) For each compact set $K$ in $R$, there is a compact set $K^{\prime}$ in $R$ which contains every bounded component of $R \backslash(F \cup K)$ whose closure meets $K$.

THEOREM 3. Let $F$ be closed in an open Riemann surface R. Consider the condition: (IV) $R^{*} \backslash F$ is connected and locally connected.

If $F$ is essentially of finite genus in $R$, then (IV) is sufficient for Runge-type harmonic approximation on $F$.

Let $G$ be an open set in $R$. We say that $(G, R)$ is a Runge pair for approximation on closed sets if for each $h$ harmonic on $G$, each subset $F$ of $G$ which is $R$-closed, and each $\varepsilon>0$, there exists a $u$ harmonic on $R$ such that $|h-u|<\varepsilon$ on $F$.

Theorem 4. Let $R$ be an open Riemann surface. In order for $(G, R)$ to be a Runge pair for harmonic approximation on closed sets, it is necessary that $R^{*} \backslash G$ be connected. If $G$ is essentially of finite genus, this condition is also sufficient.

Let $F$ be closed in an open Riemann surface $R$. We say that Walsh-type approximation by (essentially) harmonic functions is possible for the pair $(F, R)$ if every function continuous on $F$ and harmonic on $F^{\circ}$ can be approximated uniformly on $F$ by functions (essentially) harmonic on $R$. Of course, the necessary conditions for Runge approximation are all the more necessary for Walsh approximation.

THEOREM 5. Let $F$ be closed and essentially of finite genus in an open Riemann surface $R$. Suppose $F=F^{\circ-}$ and $\partial F$ is analytic. Then $F$ is a set of Walsh approximation by essentially harmonic functions. If, moreover, $R^{*} \backslash F$ is connected, then $F$ is a set of Walsh approximation by harmonic functions.

We remark that conditions (I) to (IV) are not independent of each other. More will be said on this later. For the present we feel it is better to retain all of these conditions, despite the redundancy, in order to grasp more easily the geometric relationship between $F$ and $R$.

In case $F$ is compact, our results are implicit in the work of Brelot [2] and Deny [3], at least when $R$ is a planar domain. Hence the thrust of our work is that we can now approximate on unbounded sets. Of course, from the compact theorems it follows that one can approximate uniformly on compact subsets of closed sets. But we are doing much more. For example, from Theorem 1 it follows that if $R$ is a 
plane domain, $F$ is $R$-closed, $h$ is harmonic on $F$, and $\varepsilon$ is positive, then there is a $u$ essentially harmonic on $R$ such that $|h-u|<\varepsilon$ everywhere on $F$. Hence, the approximation is uniform simultaneously on all of $F$, not just on compact subsets.

It should also be pointed out that we make no assumption about the behavior near "infinity" of the function to be approximated. It could oscillate wildly.

Such theorems are already known for holomorphic or meromorphic approximation and have borne rich fruits (see for example [3]). If $R$ is simply connected the sufficiency in Theorem 3 , for instance, is a trivial consequence of the holomorphic analogue obtained by passing to the holomorphic completion of a harmonic function. However, if $R$ is not simply connected, we see no way of deducing the harmonic results from the holomorphic analogues.

The only paper we are aware of which deals with the subject of this paper is the work by Saginjan [11]. In that paper Saginjan works in $\mathbf{R}^{n}$, but restricts his investigation to closed subsets $F$ with no interior. Thus, he is approximating continuous functions by harmonic functions.

We do not know whether our own results extend to $\mathbf{R}^{n}$, and to arbitrary open Riemann surfaces. However, the proofs would need to be modified in the case of $\mathbf{R}^{n}$ as we make use of conformal mappings. Moreover, we note that the holomorphic analogues fail on arbitrary open Riemann surfaces [5] and in $\mathbb{C}^{n}, n>1$.

ADDED IN PROOF. We have recently extended some of our results to $\mathbf{R}^{n}, n>2$.

2. Preliminaries. Let $K$ be a compact set in an open Riemann surface $R$, and let $f$ be in $C(\partial K)$, the class of continuous (real-valued) functons on $\partial K$. We may extend $f$ continuously to a neighborhood of $K$. Let $\left\{\Omega_{n}\right\}$ be a sequence of neighborhoods of $K$ nesting down on $K$ and such that each $\Omega_{n}$ is regular for the Dirichlet problem. Denote by $H^{n}(f)$ the solution of the Dirichlet problem on $\Omega_{n}$ with boundary function $f \mid \partial \Omega_{n}$. Recall that a point $x \in \partial K$ is called stable for $K$ if $H^{n}(f)(x) \rightarrow f(x)$ for each $f \in C(\partial K)$. It is well known that $x$ is stable for $K$ if and only if $R \backslash K$ is not thin at $x$ [2]. Denote by $S(K)$ the points of $\partial K$ which are stable for $K$.

LemMa 1. Let $K$ be compact and $f \in C(K)$. Then

$$
H^{n}(f) \rightarrow f
$$

uniformly on compact subsets of $S(K)$.

Since $f$ can be uniformly approximated by a difference of continuous subharmonic functions [6, p. 196], we may assume that $f$ itself is subharmonic as well as continuous. Thus, $H^{n}(f)$ decreases to $f$ on $S(K)$. The lemma now follows from Dini's theorem [6, p. 35].

Let $A$ be a subset of an open Riemann surface $R$ and denote by $\hat{A}$ the envelope of $A$. That is, $\hat{A}$ is the union of $A$ and all bounded components of $R \backslash A$. The set $A$ is called full (plein in French) in $R$ if it is equal to its envelope. If $A$ is closed, compact, or open, then $\hat{A}$ is closed, compact, or open, respectively. If $A \subset B$, then $\hat{A} \subset \hat{B}$. For references to proofs and to the historical development of this notion, see [9].

LEMMA 2. $A$ is full iff $R^{*} \backslash A$ is connected. 
Proof. Suppose first that $A$ is full, and let $V$ be a component of $R^{*} \backslash A$. It suffices to show that the ideal point $\infty$ is in $V$. Suppose $\infty \notin V$. Thus, $V$ is a component of $R \backslash A . \bar{V}$ is not compact (since $A$ is full), and so $V$ is contained in no compact set. Hence, $\infty$ is in the $R^{*}$-closure of $V$. Since $V$ is a component of $R^{*} \backslash A$, it follows that $\infty \in V$, which is the desired contradiction.

Conversely, suppose $R^{*} \backslash A$ is connected, and let $V$ be a component of $R \backslash A$. It is enough to show that $V$ is not bounded. Now $V$ is open and closed in $R \backslash A$. Thus, $V$ is open in $R^{*} \backslash A$. If $V$ were bounded, then $V$ would also be closed in $R^{*} \backslash A$. Since $R^{*} \backslash A$ is connected, $V$ would have to be all of $R^{*} \backslash A$ which is not possible since $\infty \notin V$. This proves the lemma.

Note that the properties we have discussed for $\hat{A}$ hold for more general spaces $R$ than we are considering.

Let $F$ be full in an open Riemann surface $R$. We shall say that an exhaustion

$$
R=\bigcup_{j=1}^{\infty} R_{j}
$$

of $R$ is compatible with $F$ if $F \cup R_{j}$ is full in $R$ for each $j$.

LEMMA 3. If $R^{*} \backslash F$ is connected and locally connected, then there is an exhaustion of $R$ compatible with $F$.

Proof. Let $\left\{G_{j}\right\}$ be a nested exhaustion of $R$ by bounded full open sets. Since $F$ is full, there is a $G_{j(1)}$ such that

$$
\left(\bar{G}_{j} \cup F\right)^{\wedge} \subset G_{j(1)} \cup F .
$$

Similarly, there is a $G_{j(2)}$ such that

$$
\left(\bar{G}_{j(1)} \cup F\right)^{\wedge} \subset G_{j(2)} \cup F .
$$

We choose inductively a subsequence $G_{j(k)}, j(k)<j(k+1), k=1,2, \ldots$, such that

$$
\left(\bar{G}_{j(k)} \cup F\right)^{\wedge} \subset G_{j(k+1)} \cup F
$$

Now set

$$
R_{k}=\left(\bar{G}_{j(k)} \cup F\right)^{\wedge \circ} \cap G_{j(k+1)},
$$

for $k=1,2, \ldots$ Then $\left\{R_{k}\right\}$ is an exhaustion by full bounded open sets and $F \cup \bar{R}_{k}$ is full. Hence, the exhaustion $\left\{R_{k}\right\}$ is compatible with $F$.

The following Cousin-type lemma shows that in order to approximate essentially harmonic functions, it is enough to know how to approximate harmonic functions.

Lemma 4 (Pfluger [8, P. 194]). Let $\left\{R_{j}\right\}$ be an open cover of an open Riemann surface $R$. If $p_{j}$ are essentially harmonic on $R_{j}$ and $p_{j}-p_{k}$ are harmonic on $R_{j} \cap R_{k}$ for all $j$ and $k$, one can find $p$ essentially harmonic on $R$ so that $p-p_{j}$ is harmonic on $R_{j}$ for every $j$.

For the next lemma, we shall say that a function $h$ is essentially a $C^{2}$-function on $R$ if

$$
h=p+\varphi
$$


where $p$ is essentially harmonic and $\varphi$ is a $C^{2}$-function.

LEMMA 5 (GREEN FORMULA). Suppose $D$ is a bounded domain with $C^{1}$-boundary in an open Riemann surface $R$. If $h$ is essentially $a C^{2}$-function on $\bar{D}$, then we can write

$$
h(z)=r(z)-\frac{1}{2 \pi} \iint_{D} g(\zeta, z) \Delta \varphi(\zeta) d \xi d \eta
$$

where $r$ is essentially harmonic on $D, g$ is the Green function for $D, \varphi$ is given by (1), $\zeta=\xi+i \eta$, and $\Delta \varphi(\zeta) d \xi d \eta$ is invariantly defined.

Proof. (2) is an immediate consequence of (1) and the well-known Green representation formula for $C^{2}$-functions:

$$
\varphi(z)=u(z)-\frac{1}{2 \pi} \iint_{D} g(\zeta, z) \Delta \varphi(\zeta) d \xi d \eta
$$

where $u$ is harmonic.

The compact version of our Runge-type Theorem 1 was proved for plane domains by Brelot [2] and Deny [3]. We need the analogous theorem on open Riemann surfaces.

LEMMA 6 (RUNGE-TYPE). Let $K$ be a compact subset of an open Riemann surface $R$. Then each function $h$ essentially harmonic on $K$ is the uniform limit of functions essentially harmonic on $R$. If $K$ is full in $R$ and $h$ is harmonic on $K$, we may take the approximating function to be harmonic on $R$.

Proof. The case where $K$ is full is due to Pfluger [8, p. 192] (see also [7, p. 347]). Let $h$ be essentially harmonic on $K$ and $\varepsilon>0$. Then by the Cousin-type Lemma 4, there is a function $h_{1}$ essentially harmonic on $R$ with $h_{1}-h$ harmonic on $K$. Let $G$ be a neighborhood of $K$ which is full in $R$ and bounded by analytic Jordan curves. An argument of Pfluger [8, p. 194] shows that $h_{1}-h$ can be approximated within $\varepsilon / 2$ on $K$ by a function $v_{0}$ essentially harmonic on $G$. Now by Pfluger's Runge-type theorem [8, p. 192], $v_{0}$ can be approximated within $\varepsilon / 2$ on $K$ by a function $v_{1}$ harmonic on $R$. Set $v=h_{1}-v_{1}$. Then $v$ is essentially harmonic on $R$ and approximates $h$ within $\varepsilon$. This completes the proof.

3. Fusion lemma. Alice Roth [10] first proved a fusion lemma for rational approximation. In this section we shall develop a harmonic analogue.

LEMMA 7. Suppose $\delta$ and $x_{0}$ are positive. Then, there is a positive constant $a=a\left(\delta, x_{0}\right)$ such that if $h$ is harmonic and $|h|<\varepsilon$ for $\left|x_{0}-x\right|<\delta$, then there is $a$ $C^{2}$-function $v$ such that

$$
\begin{gathered}
v=0, \text { for } x \leqslant 0, \\
|v|<a \varepsilon, \text { for } 0<x<x_{0}, \\
v=h, \text { for } x_{0} \leqslant x<x_{0}+\delta \\
|\Delta v|<a \varepsilon, \quad \text { whenever } v \text { is defined. }
\end{gathered}
$$

Proof. We shall construct $v$ of the form

$$
v(x, y)=\sum_{i=0}^{5} a_{i}(y) x^{i}, \quad 0 \leqslant x \leqslant x_{0} .
$$


The boundary conditions are

$$
\left.\begin{array}{c}
v(0, y)=v_{x}(0, y)=v_{x x}(0, y)=0, \\
v\left(x_{0}, y\right)=h\left(x_{0}, y\right), \\
v_{x}\left(x_{0}, y\right)=h_{x}\left(x_{0}, y\right), \\
v_{x x}\left(x_{0}, y\right)=h_{x x}\left(x_{0}, y\right) .
\end{array}\right\}
$$

From (1) we have $a_{0}=a_{1}=a_{2}=0$.

From (2) we have

$$
\begin{aligned}
x_{0}^{3} a_{3}(y)+x_{0}^{4} a_{4}(y)+x_{0}^{5} a_{5}(y) & =h\left(x_{0}, y\right), \\
3 x_{0}^{2} a_{3}(y)+4 x_{0}^{3} a_{4}(y)+5 x_{0}^{4} a_{5}(y) & =h_{x}\left(x_{0}, y\right), \\
6 x_{0} a_{3}(y)+12 x_{0}^{2} a_{4}(y)+20 x_{0}^{3} a_{5}(y) & =h_{x x}\left(x_{0}, y\right) .
\end{aligned}
$$

This system has a solution $a_{3}, a_{4}, a_{5}$. Each $a_{j}$ is a linear combination of $h\left(x_{0}, y\right)$, $h_{x}\left(x_{0}, y\right)$, and $h_{x x}\left(x_{0}, y\right)$.

Fix a value $y_{0}$. In the disc $D\left(y_{0}\right)$ of center $\left(x_{0}, y_{0}\right)$ and radius $\delta / 2$, the functions $h, h_{x}$, and $h_{x x}$ have integral representations obtained by differentiating the Poisson formula for $h$. Since $h$ is bounded by $\varepsilon$ in $\left|x_{0}-x\right|<\delta$, there is a constant $A$ independent of $y_{0}$ such that $h\left(x_{0}, y_{0}\right), h_{x}\left(x_{0}, y_{0}\right), h_{x x}\left(x_{0}, y_{0}\right)$ are bounded by $A \varepsilon$. Hence the $a_{j}$ are also bounded by a constant times $\varepsilon$ and the same is true for $v$ on $0<x \leqslant x_{0}$. Since the $a_{j}$ are bounded by a constant times $\varepsilon$, it also follows that the same holds for $\Delta v$.

There only remains to verify that $v$ is a $C^{2}$-function, and this need only be checked on the lines $x=0$ and $x=x_{0}$. Along these lines the partial derivatives are continuous from the left and continuous from the right and therefore continuous.

LEMMA 8. Let $x_{0}>0$ and suppose $U$ is a neighborhood of the segment $x=x_{0}$, $0 \leqslant y \leqslant y_{0}$. Then there is a positive constant $a=a(U)$ such that if $h$ is harmonic and bounded by $\varepsilon$ on $U$, then there is $a v$ as in the previous lemma, but now $v$ is only defined on

$$
(x \leqslant 0) \cup\left(0 \leqslant x \leqslant x_{0}, 0 \leqslant y<y_{0}\right) \cup\left(U \cap\left(x>x_{0}\right)\right) .
$$

The proof is identical to that of the previous lemma.

LEMMA 9. Suppose $\delta>0$ and $\rho>1$. Then there is a positive constant $a=a(\delta)$ such that if $h$ is harmonic and $|h|<\varepsilon$ for ||$z|-\rho|<\delta$, then there is a $C^{2}$-function $v$ such that

$$
\begin{gathered}
v=0, \text { for }|z|<1, \\
|v|<a \varepsilon, \text { for } 1 \leqslant|z|<\rho, \\
v=h, \text { for } \rho<|z|, \\
|\Delta v|<a \varepsilon, \quad \text { where } v \text { is defined. }
\end{gathered}
$$

Proof. The mapping $z=e^{\zeta}$ transports $h(z)$ to a function $\tilde{h}(\zeta)$ which satisfies the hypotheses of Lemma 7. Since $\tilde{h}$ is periodic, the proof of Lemma 7 furnishes a function $\tilde{v}$ which is also periodic. Thus $\tilde{v}(\zeta)$ pulls back to a function $v(z)$ which solves our problem. We note that $|\Delta v|<|\Delta \tilde{v}|$. This lemma could also have been 
proved directly by passing to polar coordinates and solving an appropriate boundary value problem.

LEMMA 10. Let $K_{1}$ and $K_{2}$ be compact subsets of a Riemann surface $R$ and let $U_{1}$ and $U_{2}$ be neighborhoods of $K_{1}$ and $K_{2}$ respectively. Then there are neighborhoods

$$
K_{j} \subset G_{j} \subset U_{j}, \quad j=1,2,
$$

and a positive constant a such that if $h$ is essentially harmonic on $U_{2}$ and bounded by $\varepsilon$ on $U_{1} \cap U_{2}$, then $h \mid G_{2}$ has an extension $\tilde{h}$ to $G_{1} \cup G_{2}$ such that $\tilde{h}$ is $C^{2}$ on $G_{1}$ and satisfies

$$
|\tilde{h}|<a \varepsilon, \quad|\Delta \tilde{h}|<a \varepsilon, \quad z \in G_{1} .
$$

Proof. For $j=1,2$, let $G_{j}$ be a neighborhood of $K_{j}$ in $U_{j}$ which is bounded by finitely many disjoint analytic Jordan curves in $U_{j}$. We may assume that $\partial G_{1}$ and $\partial G_{2}$ are transversal to each other in the sense that they have no points of tangency and the angles of intersection must be positive. Let $\tilde{G}_{2}$ be a "swelled" neighborhood of $G_{2}$ obtained by moving each boundary curve of $G_{2}$ slightly away from $G_{2}$. We may assume $\partial \tilde{G}_{2}$ is analytic. Each component of $\tilde{G}_{2} \backslash G_{2}$ is called a collar and we may assume that each collar is homeomorphic to an annulus. We may construct $\tilde{G}_{2}$ so close to $G_{2}$ that if $C$ is any collar, then the components of $\bar{C} \cap \bar{G}_{1}$ are either all of $\bar{C}$ or else disjoint quadrilaterals with one side of the quadrilateral on $\partial \tilde{G}_{2}$, the opposite side on $\partial G_{2}$, and the other two on $\partial G_{1}$. The components of $C \cap \bar{G}_{1}$ are called transition domains. We may assume that $\tilde{G}_{2}$ is so close to $G_{2}$ that each transition domain is in $U_{1} \cap U_{2}$.

We set $\tilde{h}=h$ on $G_{2}$ and $\tilde{h}=0$ on $G_{1} \backslash \tilde{G}_{2}$. Thus, we have only to define $\tilde{h}$ on the transition domains.

Let $D$ be a transition domain. Then $D$ is conformally equivalent to either an annulus or a rectangle. Suppose first that $D$ is equivalent to an annulus $(1-\delta<$ $|z|<\rho+\delta)$. Let $f$ be the conformal map. By Lemma 9 there is a constant $a_{1}$ independent of $h$ and a $C^{2}$-function $h_{1}$ satisfying

$$
\begin{gathered}
h_{1}=0, \quad \text { for }|z|<1, \\
\left|h_{1}\right|<a_{1} \varepsilon, \quad \text { for } 1<|z|<\rho, \\
h_{1}=h \circ f, \quad \text { for } \rho<|z|, \\
\left|\Delta h_{1}\right|<a_{1} \varepsilon, \quad \text { where } h_{1} \text { is defined. }
\end{gathered}
$$

Now set $\tilde{h}=h_{1} \circ f^{-1}$ on $D$. Then $\tilde{h}$ has the necessary properties and $\Delta \tilde{h}=$ $\Delta h_{1} /\left|f^{\prime}\right|^{2}$ is appropriately bounded since $f^{\prime}$ is bounded away from zero on $1<|z|$ $<\rho$. The bound $a(D)$ which we obtain depends only on $D$ and not on $h$. Thus, $\tilde{h}$ has been defined on $D$ in case $D$ is homeomorphic to an annulus.

If $D$ is not an annulus then $D$ is a "quadrilateral" with one side $\alpha_{1}$ on $\partial G_{2}$ and the opposite side $\alpha_{0}$ on $\partial \tilde{G}_{2}$. There are positive constants $x_{0}$ and $y_{0}$ and $\delta$ and a conformal map $f$ of the rectangle $\left(-\delta<x<x_{0}+\delta, 0<y<y_{0}\right)$ onto $D$ such that the left and right boundaries correspond to $\alpha_{0}$ and $\alpha_{1}$ respectively. As in the annular case, but using Lemma 8 instead, there is a function $h_{1}$ satisfying 


$$
\begin{gathered}
h_{1}=0, \quad \text { for } x<0, \\
\left|h_{1}\right|<a_{1} \varepsilon, \text { for } 0<x<x_{0}, 0<y<y_{0}, \\
h_{1}=h \circ f, \quad \text { for } x_{0}<x, 0<y<y_{0} \\
\left|\Delta h_{1}\right|<a_{1} \varepsilon, \quad \text { where } h_{1} \text { is defined. }
\end{gathered}
$$

Again, set $\tilde{h}=h_{1} \circ f^{-1}$ on $D$. To see that

$$
\Delta \tilde{h}=\Delta h_{1} /\left|f^{\prime}\right|^{2}
$$

is appropriately bounded, we have only to look at $f^{\prime}$ on

$$
Q=\left(0 \leqslant x \leqslant x_{0}, 0<y<y_{0}\right) \text {, }
$$

for $\Delta h_{1}$ is zero elsewhere. Thus, it is of no consequence that $f^{\prime}$ may approach zero. We need only the fact that $f^{\prime}$ is bounded away from zero on $Q$ for by the symmetry principle $f$ extends conformally across the boundaries

$$
\left(-\delta<x<x_{0}+\delta, y=0\right) \text { and }\left(-\delta<x<x_{0}+\delta, y=y_{0}\right) \text {. }
$$

Hence we have extended $\tilde{h}$ to each transition domain and we have found an associated constant. Let $a$ equal the maximum of these constants as we vary over the finitely many transition domains. This completes the proof.

LEMMA 11. Let $R$ be an open Riemann surface and let $K_{1}, K_{2}, U_{1}, U_{2}$ be as in the previous lemma. Then there is a positive constant a such that if $h$ is essentially harmonic on $U_{2}$ and bounded by $\varepsilon$ on $U_{1} \cap U_{2}$, then there is a function $r$ essentially harmonic on $R$ satisfying

$$
\begin{gathered}
|r|<a \varepsilon, \quad \text { on } K_{1}, \\
|r-h|<a \varepsilon, \quad \text { on } K_{2} .
\end{gathered}
$$

Proof. Let $a_{1}$ be the constant of the previous lemma. If $\tilde{h}$ is as in the previous lemma then by the Green formula, we may write

$$
\tilde{h}=r(z)-\frac{1}{2 \pi} \int_{G_{1} \cup G_{2}} g(\zeta, z) \Delta \tilde{h}(\zeta) d \xi d \eta
$$

where $g$ is the Green function for $G_{1} \cup G_{2}$ and $r$ is essentially harmonic on $G_{1} \cup G_{2}$. We set

$$
a=a_{1}+\sup _{K_{1} \cup K_{2}} a_{1} \iint_{G_{1} \cup G_{2}} g(\zeta, z) d \xi d \eta .
$$

Then $a$ and $r$ have the required properties, except that $r$ is only essentially harmonic on $G_{1} \cup G_{2}$. However, $r$ has only finitely many singularities and so by the Runge-type lemma, we may assume that $r$ is essentially harmonic on all of $R$. This proves the lemma.

At last we may state the

FUSION LEMMA 12. Let $K_{1}$ and $K_{2}$ be compact sets in an open Riemann surface $R$ and let $V$ be an open neighborhood of $K_{1} \cap K_{2}$. There is a positive constant a such that if $q_{1}$ and $q_{2}$ are essentially harmonic functions on $R$ satisfying for some $\varepsilon>0$,

$$
\left|q_{1}-q_{2}\right|_{\nu}<\varepsilon
$$


then there is a function $r$, essentially harmonic on $R$, such that for $j=1,2$,

$$
\left|r-q_{j}\right|_{K_{j}}<a \varepsilon .
$$

RemarK 1. Clearly, if $K_{1} \cup K_{2}$ is full in $R$ and if $q_{1}$ and $q_{2}$ are harmonic on $K_{1} \cup K_{2}$, we may assume that $r$ is actually harmonic on $R$.

Proof. Let $U_{1}$ and $U_{2}$ be open neighborhoods of $K_{1}$ and $K_{2}$ respectively such that $U_{1} \cap U_{2}=V$. Set $q=q_{1}-q_{2}$. Then $q$ satisfies the hypotheses of the previous lemma, and so there exists a function $s$ essentially harmonic on $R$ and satisfying

$$
|s|<a \varepsilon, \quad z \in K_{1} ; \quad|s-q|<a \varepsilon, \quad z \in K_{2} .
$$

Set $r=q_{1}-s$. Then $r$ has the required properties and the proof is complete.

4. Necessity. Before proving our theorems, we shall discuss the interdependence of some of our conditions.

RemarK 1. Conditions (II) and (III) together imply $\partial F=\partial \hat{F}$.

Proof. We observe that $\partial \hat{F} \subset \partial F$ and that $\partial F \backslash \partial \hat{F}=\partial F \cap(\hat{F})^{0}$. We show that $\partial F=\partial \hat{F}$. For suppose, to obtain a contradiction, that $p \in(\hat{F})^{0} \cap \partial F$. Since $p \in$ $(\hat{F})^{0}$ we may choose a closed bounded ball $B$ with $p \in B^{0}, B \subset \hat{F}$. Let $\alpha_{j}$, $j=1,2, \ldots$, be the (at most countably many) components of $\partial B \backslash F$. For each $j$, let $U_{j}$ be the component of $R \backslash F$ containing $\alpha_{j}$. Since $p \in(\hat{F})^{0}$, we may assume that $B$ is so small that each $U_{j}$ is bounded. We now set

$$
V=B^{0} \cup \bigcup_{j=1}^{\infty} U_{j} .
$$

From condition (III) it follows that $V$ is bounded and clearly $\partial V \subset F$. Thus $V$ violates condition (II), which is the desired contradiction. We have shown that $\partial F=\partial \hat{F}$.

REMARK 2. (III) $\rightarrow$ (I).

From the previous remarks, we have only to show the necessity of (III) and (II) for Theorem 2.

Proof of NeCESSITY of (II). Suppose $V$ is a bounded open set such that $\partial V \subset F$, $x_{1} \in V \cap F$ and $x_{0} \in V \backslash F$. We may assume that $V$ is connected. The sufficiency proofs to come (which generalize Brelot-Deny and which are independent of necessity proofs) show there is a harmonic $f$ on $R \backslash\left\{x_{0}\right\}$ which is less than 0 on $\partial V$ and more than 2 at $x_{1}$. Suppose $f$ can be approximated within 1 on $F$ by $h$ harmonic on $R$. Then $h$ violates the maximum principle in $V$. This proves the necessity of (II).

To complete the necessity in Theorem 2, we have only to show condition (III) is necessary. Suppose then that approximation is possible, and to obtain a contradiction, we suppose that condition (III) fails. Then for some compact $K \subset R$, $R \backslash(F \cup K)$ has bounded components which reach arbitrarily far out. To be more precise, we may exhaust $R$ by full domains $G_{n}$ in such a way that for $n=$ $1,2, \ldots$,

$$
\bar{G}_{n} \subset G_{n+1},
$$


and $(R \backslash F) \backslash \bar{G}_{1}$ has components $D_{n}$, with

$$
D_{n} \cap G_{2} \neq \varnothing, \quad D_{n} \subset G_{5 n}, \quad D_{n} \not G_{5 n-1} \text {. }
$$

For $n=3,4, \ldots$, choose

$$
a_{n} \in D_{n} \cap \partial G_{2}, \quad b_{n} \in D_{n} \cap \partial G_{5 n-1} .
$$

We may assume that $a_{n}$ converges to some point $a \in \partial G_{2}$. Of course $b_{n}$ tends to the ideal boundary.

Let $U_{n}$ be the component of $D_{n} \backslash \bar{G}_{5 n-4}$ which contains $b_{n}$. Also, let $\omega_{n}$ be the harmonic measure for $D_{n}$ at $a_{n}$, considered as a measure on $\partial D_{n}$. We shall construct a sequence $f_{n}$ with the following properties:

$$
\begin{gathered}
f_{n} \text { is harmonic on } R \backslash b_{n}, \\
\left|f_{n}(p)\right|<2^{-n}, \quad p \in \bar{G}_{5 n-5}, \\
\sum_{j=1}^{n} f_{j}(p)>2\left(n+\left\|\sum_{j=1}^{n-1} f_{j}\right\|_{D_{n}}\right) \omega_{n}\left(\partial U_{n} \backslash G_{5 n-1}\right)^{-1},
\end{gathered}
$$

for $p \in E_{n}$, where $E_{n}$ is some subset of $\partial U_{n} \backslash G_{5 n-1}$ whose $\omega_{n}$-measure exceeds

$$
\begin{gathered}
\omega_{n}\left(\partial U_{n} \backslash G_{5 n-1}\right) / 2 . \\
f_{n}(p) \geqslant 0, \quad p \in \partial \bar{D}_{n} .
\end{gathered}
$$

Suppose for the moment that such a sequence $f_{n}$ has been constructed. Then by (2), $\Sigma f_{n}$ converges to some $f$ harmonic on $F$. Suppose, to obtain a contradiction, that there is a $g$ harmonic on $R$ with $\|f-g\|_{F}<1$. Then

$$
\begin{aligned}
g\left(a_{n}\right)= & \int_{\partial D_{n}} g d \omega_{n}=\int_{\partial D_{n}}(g-f) d \omega_{n}+\int_{\partial D_{n}} f d \omega_{n} \\
> & \int_{\partial D_{n}} f d \omega_{n}-1=\int_{E_{n}} f d \omega_{n}+\int_{\partial D_{n} \backslash E_{n}} f d \omega_{n}-1 \\
> & \int_{E_{n}} \sum_{1}^{n} f_{j} d \omega_{n}+\int_{E_{n}} \sum_{n+1}^{\infty} f_{j} d \omega_{n}+\int_{\partial D_{n} \backslash E_{n}} \sum_{1}^{\infty} f_{j} d \omega_{n}-1 \\
> & 2\left(n+\left\|\sum_{1}^{n-1} f_{j}\right\|_{D_{n}}\right) \omega_{n}\left(\partial U_{n} \backslash G_{5 n-1}\right)^{-1} \omega_{n}\left(\partial U_{n} \backslash G_{5 n-1}\right) / 2 \\
& -\frac{1}{2^{n}}-\left\|\sum_{1}^{n-1} f_{j}\right\|_{D_{n}}+\int_{\partial D_{n} \backslash E_{n}} f_{n} d \omega_{n}-\frac{1}{2^{n}}-1>n-2 .
\end{aligned}
$$

We have seen that $g\left(a_{n}\right) \geqslant n-2$, and since $a_{n}$ converges to $a$, this contradicts the harmonicity of $g$ at $a$. Hence, the proof is complete, modulo the construction of the sequence $\left\{f_{n}\right\}$.

Set $f_{1}=0$, and suppose $f_{j}$ have been constructed for $j=1,2, \ldots, n-1$. Let $A_{n}$ be the envelope of $\bar{G}_{5 n-4} \cup \partial U_{n}$ with respect to $R \backslash\left\{b_{n}\right\}$. Now set

$$
K_{n}=\bar{G}_{5 n-4} \cup \partial \hat{A}_{n}=\bar{G}_{5 n-4} \cup \alpha_{n} \text {, }
$$


where $\alpha_{n}$ is part of $\partial U_{n} \backslash \bar{G}_{5 n-4}$. We define $\varphi=\varphi_{n}$ on $K_{n}$ as follows:

$$
\varphi=2\left(n+\left\|\sum_{1}^{n-1} f_{j}\right\|_{D_{n}}\right) \omega_{n}\left(\alpha_{n} \backslash G_{5 n-1}\right)^{-1}+\left\|\sum_{1}^{n-1} f_{j}\right\|_{D_{n}}+2,
$$

on $\alpha_{n} \backslash G_{5 n-1}$.

$$
\varphi=2^{-(n+1)}, \quad \text { on } K_{n} \cap G_{5 n-2} .
$$

(7) $\varphi$ is extended continuously to the rest of $\alpha_{n}$ (and hence to $K_{n}$ ) without changing its upper and lower bounds.

The function $\varphi$ is continuous on $K_{n}$ and harmonic on $K_{n}^{0}$.

We claim that $\partial K_{n}$ is stable. Indeed, we have only to verify that the complement of $K_{n}$ is not thin at any boundary point of $K_{n}$. This follows from [6, p. 216] and since $K_{n}$ is full in $R \backslash\left\{b_{n}\right\}$.

Since $\partial K_{n}$ is stable, we may approximate $\varphi$ by a function harmonic on $K_{n}$ (Lemma 1), and since $K_{n}$ is full in $R \backslash\left\{b_{n}\right\}$, we may even, according to the Pfluger-Runge theorem (Lemma 6), approximate by a function $f_{n}$ harmonic on $R \backslash\left\{b_{n}\right\}$. We may approximate so well that $f_{n}$ satisfies (1), (2), (3), and (4), and the proof of necessity is complete for Theorem 2 .

To prove the necessity in Theorem 4, suppose $R^{*} \backslash G$ is not connected. Then there is a compact curve $\gamma$ bounding an open set $V$ which contains a point of $G$ and a point of $R \backslash G$. Then the proof of necessity of (II) now applies.

5. Sufficiency. We shall prove simultaneously Theorem 1 and the sufficiency in Theorem 3.

For any of our theorems we introduce the special case of that theorem. By the special case of Theorem $n$ we mean Theorem $n$ restricted to pairs $(F, R)$ such that $R$ has an essential extension in which $F$ is bounded. We shall now show that in order to prove Theorem 1 and the sufficiency in Theorem 3, it is sufficient to do so for the special cases.

Suppose, then, that the special cases of Theorem 1 and the sufficiency in Theorem 3 have already been established and let $h$ be essentially harmonic on $F$. Let $\left\{M_{j}\right\}$ be a locally finite cover of $F$ by disjoint open sets of finite genus. We may assume $h$ is defined on each $M_{j}$. Let $\left\{R_{n}\right\}$ be an exhaustion of $R$ with the property that

$$
R_{j} \cap M_{k}=\varnothing, \quad k \geqslant j .
$$

If $R^{*} \backslash F$ is connected and locally connected, we may further assume that the exhaustion is compatible with $F$.

Let $h$ be essentially harmonic on $F$. Since $R_{1} \cup M_{1}$ has finite genus, it has a compact extension by a theorem of Bochner [1]. Thus, if $\varepsilon>0$, then by the special case of Theorem 1 , there is a $u_{1}$ essentially harmonic on $R_{1} \cup M_{1}$ such that

$$
\left|u_{1}-h\right|_{F \cap M_{1}}<\varepsilon / 2
$$

Moreover, if $R^{*} \backslash F$ is connected and locally connected and if $h$ is harmonic on $F$, then by the special case of Theorem 3, we may take $u_{1}$ to be harmonic on $R_{1} \cup M_{1}$.

Set $R_{0}=\varnothing$. Again by the special case of Theorem 1 , there is a $u_{2}$ essentially 
harmonic on

$$
R_{2} \cup \bigcup_{j=1}^{2} M_{j}
$$

such that

$$
\left|u_{2}-u_{1}\right|<\varepsilon / 2^{2}, \quad \text { on } \bar{R}_{0} \cup\left(F \cap M_{1}\right)
$$

and

$$
\left|u_{2}-h\right|<\varepsilon / 2^{2} \text {, on } F \cap M_{2} .
$$

We proceed inductively to construct $u_{n}, n=2,3, \ldots$, essentially harmonic on

$$
R_{n} \cup \bigcup_{j=1}^{n} M_{j}
$$

such that

$$
\left|u_{n}-u_{n-1}\right|<\varepsilon / 2^{n}, \quad \text { on } \bar{R}_{n-2} \cup\left\{F \cap \bigcup_{j=1}^{n-1} M_{j}\right\} \text {, }
$$

and

$$
\left|u_{n}-h\right|<\varepsilon / 2^{n}, \quad \text { on } F \cap M_{n} \text {. }
$$

Note that $u_{n}$ converges to a function $u$ essentially harmonic on $R$, and that

$$
|u-h|<\varepsilon / 2^{n-1} \text {, on } F \cap M_{n} \text {. }
$$

Thus, our approximation is actually somewhat better than uniform. In the context of Theorem 3, we may assume that each $u_{n}$ is harmonic so that $u$ is also. This completes the proof of Theorem 1 and the sufficiency in Theorem 3 modulo the special cases.

Before proving the special cases, we note that we may assume $h$ is actually harmonic on $F$, for by the Cousin-type theorem, there is a function $h_{1}$, essentially harmonic on $R$, such that $h_{1}-h$ is harmonic on $F$. Suppose we can find $u_{1}$ essentially harmonic on $R$ such that $u_{1}$ approximates $h-h_{1}$ on $F$. Then $u_{1}+h_{1}$ approximates $h$.

To prove the special cases, suppose $R$ has an essential extension $R^{\prime}$ in which $F$ is bounded. Let $h$ be harmonic on $F$ and fix $\varepsilon>0$. We may assume that $h$ is harmonic on a (possibly infinite) polygonal neighborhood $U$ of $F$. We may assume that if $R^{*} \backslash F$ is connected and locally connected, the same holds for $R^{*} \backslash \bar{U}$ (see [4, p. 152]). Let $\left\{R_{n}\right\}$ be an exhaustion of $R$. If $R^{*} \backslash \bar{U}$ is connected and locally connected, we may assume that $\left\{R_{n}\right\}$ is compatible with $\bar{U}$. For each $n$, set $U_{n}=U \cap R_{n}$. Let $\bar{F}$ be the $R^{\prime}$-closure of $F$ and let $\tilde{R}$ be an open Riemann surface with $R \cup \bar{F} \subset \tilde{R} \subset R^{\prime}$. We apply the Fusion Lemma to the Riemann surface $\tilde{R}$, replacing $K_{1}, K_{2}, V$ respectively by $\bar{R}_{n}, \bar{F} \backslash R_{n}, U_{n+1}$. We may choose the $\left\{a_{n}\right\}$ so that $1<a_{n}<a_{n+1}$. We select the positive numbers $\varepsilon_{1}, \varepsilon_{2}, \ldots$, so that

$$
\varepsilon_{n+1}<\varepsilon_{n} \text { and } \sum_{n=1}^{\infty} \varepsilon_{n}<\varepsilon / 2 \text {. }
$$


By Lemma 6, there exist essentially harmonic functions $q_{n}$ on $\tilde{R}$ such that

$$
\left|q_{n}-h\right|<\varepsilon_{n} / 2 a_{n} \text {, on } \bar{U}_{n+1},
$$

and therefore,

$$
\left|q_{n+1}-q_{n}\right|<\varepsilon_{n} / a_{n}, \quad \text { on } \bar{U}_{n+1}, n=1,2, \ldots
$$

Moreover, if $R^{*} \backslash \bar{U}$ is connected and locally connected, the same is true of $\tilde{R}^{*} \backslash \bar{U}$ and we may choose each $q_{n}$ to be harmonic. By the Fusion Lemma, for each $n=1,2, \ldots$, there exists an essentially harmonic function $r_{n}$ on $\tilde{R}$ such that

$$
\begin{array}{cc}
\left|r_{n}-q_{n}\right|<\varepsilon_{n}, & \text { on } \bar{R}_{n}, \\
\left|r_{n}-q_{n+1}\right|<\varepsilon_{n}, & \text { on } \bar{F} \backslash R_{n} .
\end{array}
$$

Moreover, if $R^{*} \backslash \bar{U}$ is connected and locally connected, we may assume that each $r_{n}$ is harmonic. The inequalities (2) yield

$$
\sum_{n}^{\infty}\left|r_{\nu}-q_{\nu}\right|<\sum_{n}^{\infty} \varepsilon_{\nu}, \quad \text { on } \bar{R}_{n}
$$

Therefore,

$$
u=q_{1}+\sum_{1}^{\infty}\left(r_{\nu}-q_{\nu}\right)
$$

is essentially harmonic on $R=\cup_{n=1}^{\infty} R_{n}$.

Set $F_{n}=F \cap \bar{R}_{n}$. From (1) and (2), there follows on $F_{1}$,

$$
|u-h| \leqslant\left|q_{1}-h\right|+\sum_{1}^{\infty}\left|r_{\nu}-q_{\nu}\right|<\frac{\varepsilon_{1}}{2 a_{1}}+\sum_{1}^{\infty} \varepsilon_{\nu}<\varepsilon .
$$

From (3), (1), and (2), we also have

$$
\begin{aligned}
|u-h| & <\sum_{1}^{n-1}\left|r_{\nu}-q_{\nu+1}\right|+\left|q_{n}-h\right|+\sum_{n}^{\infty}\left|r_{\nu}-q_{\nu}\right| \\
& <\sum_{1}^{n-1} \varepsilon_{\nu}+\frac{\varepsilon_{n}}{2 a_{n}}+\sum_{n}^{\infty} \varepsilon_{\nu}<\varepsilon
\end{aligned}
$$

on $F_{n} \backslash F_{n-1}, n=2,3, \ldots$

Thus, $u$ can be approximated uniformly on $F$ by functions essentially harmonic on $R$, and by harmonic functions in case $R^{*} \backslash F$ is connected and locally connected. This completes the proof of Theorem 1 and the proof of sufficiency in Theorem 3.

Let us now prove the sufficiency in Theorem 4. Actually this is a corollary of the sufficiency in Theorem 3. Indeed, suppose $G$ is open in the open Riemann surface $R$, that $G$ is essentially of finite genus, and that $R^{*} \backslash G$ is connected. Let $h$ be harmonic on $G$; let $F$ be a closed subset of $R$ contained in $G$; and let $\varepsilon>0$. We may construct a locally finite, closed, polygonal neighborhood $P$ of $F$ in $G$ with $R^{*} \backslash P$ connected. Since $\partial P$ is locally finite, it is clear that $R^{*} \backslash P$ is also locally connected. From Theorem 3 , there is a function $u$ harmonic on $R$ such that $|u-h|<\varepsilon$ on $P$. This completes the proof of sufficiency in Theorem 4 . 
We shall now prove the sufficiency in Theorem 5. Let $h$ be continuous on $F$ and harmonic on $F^{\circ}$. Let $\left\{p_{n}\right\}$ be chosen, one from each component of $R-F$. Since $\partial F$ is analytic, $\left\{p_{n}\right\}$ is closed. Set $\tilde{R}=R \backslash \cup_{n=1}^{\infty}\left\{p_{n}\right\}$. Then $\partial F$ satisfies the hypotheses of Saginjan's theorem [11] in $\tilde{R}$. We note that Saginjan's theorem holds also on open Riemann surfaces. Thus, there is a harmonic function $v$ on $\tilde{R}$ such that

$$
|v-h|<\varepsilon / 2 \text { on } \partial F
$$

where $\varepsilon$ is a prescribed positive number. Let $\boldsymbol{H}_{v-h}$ denote the Perron-Wiener-Brelot solution of the Dirichlet problem for $F^{\circ}$ with boundary values $u-h$. Then, on $F$, $-\varepsilon / 2 \leqslant H_{v-h} \leqslant \varepsilon / 2$. Let $u=v-h-H_{v-h}$. Then $u=0$ on $\partial F$. Assuming that $\partial F$ is analytic, $u$ extends to a harmonic function on $F$ which we shall continue to denote by $u$. Hence, we are back in the Runge case and therefore there exists an essentially harmonic function $r$ on $R$ such that

$$
|u-r|<\varepsilon / 4 \text { on } F \text {. }
$$

If $R^{*} \backslash F$ is connected, we may take $r$ to be harmonic on $R$. Thus, on $F$ we have

$$
\begin{aligned}
|(v-r)-h| & =|\{(v-u)-h\}+(u-r)| \\
& \leqslant|(v-u)-h|+|u-r|=\left|H_{v-h}\right|+|u-r| \\
& <\varepsilon / 2+\varepsilon / 4=3 \varepsilon / 4 .
\end{aligned}
$$

If $R^{*} \backslash F$ is connected, $v-r$ is the required harmonic function on $R$ which approximates $h$. If $R^{*} \backslash F$ is not connected, we may, by Theorem 1 , approximate $v$ within $\varepsilon / 4$ on $F$ by a function $v_{1}$ essentially harmonic on $R$. In this case, $v_{1}-r$ yields the required approximation.

This paper has been concerned mostly with Runge-type approximation. We included Theorem 5 on Walsh-type approximation because it is easily derived from the Runge-type theorem. The assumptions in Theorem 5 can be somewhat relaxed, but this will be discussed in a subsequent paper dealing directly with Walsh-type approximation.

\section{REFERENCES}

1. S. Bochner, Fortsetzung Riemannscher Flächen, Math. Ann. 98 (1928), 406, 421.

2. M. Brelot, Sur l'approximation et la convergence dans la théorie des fonctions harmoniques ou holomorphes, Bull. Soc. Math. France 73 (1945), 55-70. MR 7, 205.

3. J. Deny, Systemes totaux de fonctions harmoniques, Ann. Inst. Fourier (Grenoble) 1 (1949), 103-113 (1950).

4. P. M. Gauthier, Meromorphic uniform approximation on closed subsets of open Riemann surfaces, Approximation Theory and Functional Analysis (J. B. Prolla, Editor), North-Holland, Amsterdam, 1979, pp. 139-158.

5. P. M. Gauthier and W. Hengartner, Approximation sur les fermés par des fonctions analytiques sur une surface de Riemann, C. R. Acad. Bulgare Sci. 26 (1973), 731-732. MR 49 \#5363.

6. L. L. Helms, Introduction to potential theory, Wiley, New York, 1969. MR 41 \#5638.

7. N. S. Landkof, Foundations of modern potential theory, Springer-Verlag, Berlin and New York, 1972.

8. A. Pfluger, Theorie der Riemannschen Flächen, Springer-Verlag, Berlin and New York, 1957.

9. A. de la Pradelle, Approximation et caractère de quasianalycité dans la théorie axiomatique des fonctions harmoniques, Ann. Inst. Fourier (Grenoble) 17 (1967), 383-399. MR 37 \# 3040. 
10. Alice Roth, Uniform and tangential approximations by meromorphic functions on closed sets, Canad. J. Math. 28 (1976), 104-111.

11. A. A. Saginjan, Uniform and tangential harmonic approximation of continuous functions on arbitrary sets, Math. Notes 9 (1971), 78-84. MR 45 \#2375.

12. S. Scheinberg, Uniform approximation by functions analytic on a Riemann surface, Ann. of Math. (2) 108 (1978), 257-298.

Départemant de Mathégatique, Université de Montréal, Montréal, Québec, Canada

Departmient of Mathematics, Arizona State University, Tempe, Arizona 85281

Department of Mathematics, Michigan State University, East Lansng, Michigan 48823 